\title{
Regional frequency analysis of extreme storm surges using the extremogram approach
}

\author{
Marc Andreevsky ${ }^{1}$, Yasser Hamdi ${ }^{2}$, Samuel Griolet ${ }^{3}$, Pietro Bernardara ${ }^{4}$, and Roberto Frau ${ }^{1}$ \\ ${ }^{1}$ LNHE, EDF R \& D, Chatou, France \\ ${ }^{2}$ Institute for Radiological Protection and Nuclear Safety, Fontenay-Aux-Roses, France \\ ${ }^{3}$ Polytech Lyon, Rochetaillée-sur-Saône, France \\ ${ }^{4}$ EDF Energy R \& D, UK Center, SW1E5JL, London, UK
}

Correspondence: Marc Andreevsky (marc.andreevsky@edf.fr)

Received: 22 August 2019 - Discussion started: 30 September 2019

Revised: 8 May 2020 - Accepted: 13 May 2020 - Published: 10 June 2020

\begin{abstract}
To withstand coastal flooding, protection of coastal facilities and structures must be designed with the most accurate estimate of extreme storm surge return levels (SSRLs). However, because of the paucity of data, local statistical analyses often lead to poor frequency estimations. The regional frequency analysis (RFA) reduces the uncertainties associated with these estimations by extending the dataset from local (only available data at the target site) to regional (data at all the neighboring sites including the target site) and by assuming, at the scale of a region, a similar extremal behavior. In this work, the empirical spatial extremogram (ESE) approach is used. This is a graph representing all the coefficients of extremal dependence between a given target site and all the other sites in the whole region. It allows quantifying the pairwise closeness between sites based on the extremal dependence. The ESE approach, which should help with have more confidence in the physical homogeneity of the region of interest, is applied on a database of extreme skew storm surges (SSSs) and used to perform a RFA.
\end{abstract}

\section{Introduction}

To resist flooding hazard in coastal areas, considering the most accurate frequency estimates of extreme storm surge return levels (SSRLs) (1000-year return level, for instance) with an appropriate confidence level becomes a major operational concern when designing protections. The $T$-year return level can be defined as a high quantile for which the probability that an extreme value (the annual maximum, for instance) exceeds this quantile is $1 / T$. When performing a local frequency analysis, the size of the sample is often too low to obtain accurate estimations of high SSRLs. For example, storm surge on-site records calculated from tidal gauge signals are usually shorter than 30 years. The associated uncertainties can be reduced by a regional frequency analysis (RFA), which attempts to exploit the similarities between sites and deals with the estimation of hydrological characteristics at sites where few or even no data are available. The RFA introduced by Dalrymple (1960) is based on the index flood method under the assumption that within a homogeneous region, extremes (normalized by a local index) are drawn from a common regional distribution. The grouping of sites into homogeneous regions defines how to exploit regional information and, thus, can have a significant impact on final results. Numerous papers have tried to tackle this issue in hydrology by studying the explanatory variables representative of the phenomena of interest (e.g., GREHYS, 1996a, b; Hosking and Wallis, 1993, 1997; Chebana and Ouarda, 2008; Das and Cunnane, 2011). The index flood concept with the approach developed by Hosking and Wallis (1993, 1997) was extensively applied in the literature, especially in hydrology to characterize river flood hazards (e.g., Hosking and Wallis, 1997). In order to characterize coastal hazards and to estimate extreme skew storm surges (SSSs), a RFA has been recently applied (e.g., Bardet et al., 2011; Bernardara et al., 2011). For convenience, we would like to recall here the definition of a skew surge: it is the difference between the maximum observed water level and the maximum predicted 
tidal level regardless of their timing during the tidal cycle (a tidal cycle contains one skew surge). Other authors recommended the use of meteorological information to delineate homogeneous regions and to carry out a RFA of rainfall (e.g., Gabriele and Chiaravalloti, 2013). The criteria of merging sites in a homogeneous region were mainly based on statistical arguments, thereby excluding physical considerations. For example, by using data from 18 sites located on the western French coasts, Bernardara et al. (2011) used a statistical test of regional homogeneity to form the whole area of interest.

More recently, Weiss (2014) introduced a physically based method to delineate homogeneous regions in order to perform a RFA of the extreme SSSs. This method depends on the storm footprints identified through a declustering algorithm using a storm propagation probabilistic criterion. However, if a target site is very close to the limit of the region, the information at the site located on the other side of the region of interest can be wrongly excluded, even though both sites likely offer similar information and have likely similar asymptotic properties. This problem is also known as the "border effect". For instance, in the regions of interest obtained by Weiss (2014), the two French sites, Boulogne-Sur-Mer and Calais, are located in two different regions, while they are geographically close, with a distance of about $30 \mathrm{~km}$. Indeed, despite the fact that both sites face different seas (North Sea and English Channel), they have the same climate (according to the climate comparator proposed by Météo-France: http://www.meteofrance. com/climat/comparateur, last access: 9 June 2020). One can also notice that there are several other areas in which sites with similar statistical and physical behavior are located on both sides of a regional border. Moreover, very distant sites can be gathered in the same region, which could involve traces of heterogeneity, even in a region considered statistically homogeneous. Acreman and Wiltshire (1987) have suggested that the sites located near the border between two regions be considered partly owned by each region. However, Burn (1990) suggests that there is no need to define boundaries between regions, and a particular region can be defined for each site (which consists of sites similar to the site of interest in terms of extremes).

To address this limitation (the border-effect problem) and to form a physically homogeneous region centered on a target site, we take up, in the present paper, an approach, which was proposed by Hamdi et al. (2016), using the empirical spatial extremogram (ESE), in which the extremal dependence between two observation series becomes a measure of the neighborhood between the two associated sites. A pairwise measure between sites based on the spatial extremal coefficients was defined to carry out a RFA applied on extreme SSSs. The composition of regions built here is based on the similarity of sites' attributes. The higher the value of the spatial extremal coefficient between the target site and another site is, the greater the dependency of extreme SSSs, therefore indicating that storms impacting the target site tend to also impact the other site, which can be included in the region of the target site. Indeed, in a region of interest, the process generating storms and impacting the target site will tend to impact the other sites in the region as well and vice versa. It is with this in mind that the processes generating storms in a region are considered physically homogeneous. Then, it is assumed that sites with sufficiently high spatial extremal coefficients (with the target site) may be included in the same region of influence of the target site (the physically homogeneous region). The region may also be considered a typical storm footprint in the neighborhood of the target site. Obviously, the dependence between sites must be taken into account in the statistical analysis. Once a physically homogeneous region (centered on the target site) is formed, the statistical homogeneity is then checked and the regional frequency estimation (and, in particular, the dependence model and the way to calculate the effective duration of observations) is subsequently performed.

Our objective in this work is to conduct a RFA using the ESE. The ESE approach should enable us to get rid of the border effect and the distance impact and also to be more confident about the physically homogenous aspect of the region. The paper is organized as follows. A description of the methods is presented in Sect. 2. The case study with SSSs data in the whole region is also presented in Sect. 2. In Sect. 3, the ESE is applied to some target sites. The results of the analysis are further discussed in Sect. 4, before the conclusion and perspectives in Sect. 5.

\section{Materials and methods}

\subsection{The empirical spatial extremogram (ESE)}

The objective of the present section is to use an approach based on the spatial extremogram to form a homogenous region to be used in a RFA of extreme SSSs. The region of interest which is expected to be centered on a given target site must be physically and statistically homogeneous. The use of the spatial extremogram technique in index-flood-based RFA is the main contribution of the present paper. It is expected that the spatial-extremogram-based procedure leads to regions of interest with no border effect and with less residual heterogeneity.

Let $X$ and $Y$ be the random explanatory variables of the SSSs at sites $S_{1}$ (the target site) and any other site $S_{2}$, respectively. Let $q_{1}$ and $q_{2}$ be the two samples extreme quantiles (thresholds above which SSSs are considered to be extremes) for sites $S_{1}$ and $S_{2}$, respectively. Site $S_{2}$ is inside the physically homogeneous region centered on the target site $S_{1}$ if, at least, a certain part of extreme SSSs from each site are likely to be simultaneously induced by the same storms. This means that there is an extremal dependency between both sites. In the spatial and pairwise dependence description the tempo- 
ral dimension should be included because storm conditions can last several days. The ESE computation is then based on the pairwise extremal dependences (between any site and the target site), and it can be defined by the spatial extremal coefficient $\rho(X, Y)$, as proposed by Hamdi et al. (2016), and expressed as

$$
\rho: R \times R \rightarrow[0,1] \quad \rho(X, Y)=\lim _{n \rightarrow \infty}\left(P\left[X \geq q_{1} \mid Y \geq q_{2}\right]\right) .
$$

The empirical and spatial extremal coefficient is the natural estimator $\widehat{\rho}$ of $\rho$, and it is defined by

$$
\hat{\rho}(X, Y)=\frac{\sum_{t=1}^{D} I\left\{X(t)>q_{1} \& Y(t)>q_{2}\right\}}{\sum_{t=1}^{N} I\left\{X(t)>q_{1}\right\}},
$$

where $I\{f\}$ is equal to 1 if $f$ is true, and to 0 otherwise, $N$ is the number of extreme events $X(t)>q_{1}$ at the target site (events that exceeded the sample extreme quantile $q_{1}$ ), and $D$ is the number of overlapped extreme twins $X(t)>$ $q_{1} \& Y(t)>q_{2}$, extreme events generated by the same storm that occurred at sites $S_{1}$ and $S_{2}$. It was considered that two observations were generated by the same storm if the time difference between their two moments of occurrence was less, in absolute value, than $6 \mathrm{~h}$ (other durations were tested, 12 and $24 \mathrm{~h}$, but the results were similar). It is noteworthy that $D$ must be large enough in order for the ESE computation to be reliable. Indeed, the larger it is, the more significant the probability of extremal dependence. The fact that $\hat{\rho}(X, Y)$ ranges between 0 and 1 indicates the existence of a certain extremal dependency between $X$ and $Y$. The higher the empirical extremal coefficient $\widehat{\rho}$, the stronger the dependency is between both sites. Indeed, we obtain an extremal coefficient equal to 1 in the case of perfect dependency between sites (theoretically, this can only happen when computing the extremal dependency between a site and itself). But more interestingly, $\widehat{\rho}$ is relatively high when a storm is observed at site $S_{1}$; thus, relatively often, this storm is also observed at site $S_{2}$. However, the extremal coefficient tends towards 0 when the site of interest is far enough from the target site. In other words, the observations at sites, generally widely separated geographically, can be considered to be asymptotically independent. An illustrative example on how the extremal dependence coefficient is empirically computed is presented in Fig. 1. As is shown in this example, among the seven extreme twins $(N=7)$, only four overlapped with the target-site extreme storm surges $(D=4)$. The extremal coefficient is then equal to 0.57 . Obviously, this is just an illustration, and as was mentioned earlier in this section, the number of extreme twins $N$ and overlapped ones $D$ must be large enough.

The scheme for obtaining the extremal dependence between a target site and all the other sites has to be applied differently for each case study. The first question one can ask is the following: from which value of the extremal dependence coefficient can two sites begin to be considered neighbors? This leads to some other questions: when does the extremal correlation begin to be statistically significant? Or from which value of $\rho_{0}$ do we begin to have a positive association between two sites (when they experience a significant simultaneous rise in water during a storm)? Objective answers to these questions cannot in any case suggest an exact value or a statistic under or above which things are different, but there is an area in the space of the parameter or a range of values that can be explored and, depending on the sensitivity to this coefficient conclusions, can be drawn.

Let $\rho_{0}$ be the threshold above which the probability of extremal dependence between $X$ and $Y$ is high enough to consider $S_{1}$ and $S_{2}$ inside the same physically homogeneous region. First and foremost, there is a trade-off associated with the selection of this threshold: a large value of $\rho_{0}$ can result in a homogeneous region that is too small, but the opposite is likely to cause a residual probability (nothing other than a noise) which interferes with the construction of the region. The threshold $\rho_{0}$ can be estimated by analyzing the pairwise extremal dependence probability (between all sites and the target site). This procedure allows for the evaluation of the maximum-residual-noise order of magnitude. In addition, the determination of "an optimal" $\rho_{0}$ has to allow for the data merging of the neighboring sites of the target site (which have an extreme dependence on it) and put aside the "false" neighbors (sites having a residual extremal dependence probability with the target site considered to be a noise). The empirical quantiles $q_{1}$ and $q_{2}$ are set in order to extract, in $X$ and $Y$ series, only extreme events per year, which allows for the computation of the empirical spatial extremal coefficient from the biggest storms of each year. We know intuitively that the threshold $\rho_{0}$ may vary depending on the climate of the region and other physical and physiographic factors such as the bathymetry and the presence or lack of presence of a tidal estuary. Finally, the target-site neighborhood contains all sites which have a probability of extremal dependence greater than $\rho_{0}$. From a physical point of view, this means that when a storm affects a given target site, it will likely impact (not systematically) only sites enclosed in the region of interest and vice versa. The neighborhood of the target site can also be considered the region of influence around the target site as introduced by Burn (1990).

\subsection{Independent storm extraction and construction of the regional frequency model}

Once the homogeneous region of interest centered on the target site is obtained, the procedure begins by constructing a regional sample of independent storms. A storm is defined as a physical event that induces extreme SSSs (i.e., exceeds the extreme quantile $q_{p}$ ) in at least one site in the region of interest. To extract independent extreme SSSs, only the maximum value is kept. 


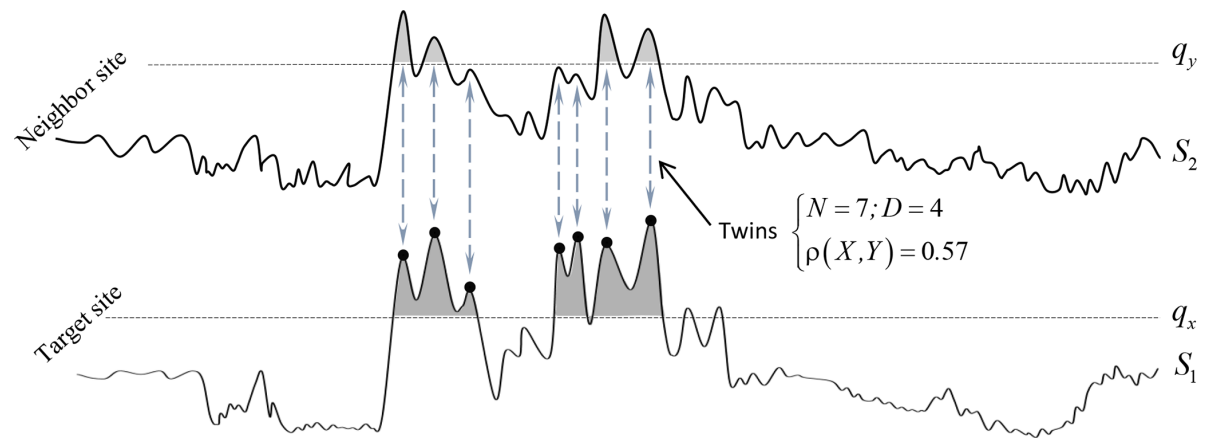

Figure 1. An illustrative example on how the extremal dependence coefficient is empirically computed. Among the seven extreme twins $(N=7)$, only four overlapped with the target-site extreme storm surges $(D=4)$. The extremal coefficient is then equal to 0.57 .

The RFA uses the flood index principal, which stipulates that within a statistically homogeneous region, extreme events normalized with a local index are drawn from a common regional distribution. It is assumed that the distribution of these extreme normalized SSSs converges to a generalized Pareto distribution (GPD) and the number of exceedances converges to a Poisson distribution. The annual SSS quantile was used as a local index to normalize on-site samples. A further noteworthy statistical setting of the developed RFA is that it uses a relatively high threshold, allowing the selection of extreme storms corresponding to an annual rate $\lambda$ of extreme SSSs equal to 1 . To meet and satisfy the statistical homogeneity requirement of the region of interest, two L-moment-based tests introduced by Hosking and Wallis (1997) are used: (i) the heterogeneity indicator $H$, which is a measure of whether the dispersion between sites is similar to a value that would be expected in a statistically homogeneous region, and (ii) the discordancy criterion $D_{\mathrm{c}}$ to ensure that any site is not significantly different, in terms of L moments, from the other sites. Hosking and Wallis (1997) suggest that a site is discordant if $D_{\mathrm{c}}<3$. In addition, a region is considered "acceptably homogeneous" if $H<1$, "possibly heterogeneous" if $1 \leq H<2$, and "definitely heterogeneous" if $H \geq 2$. These tests are performed for each region of interest. A site is generally eliminated from the inference if it is found to be discordant. To make the best possible use of the expertise (to be more conservative, for instance), the results are compared with and without the inclusion of discordant sites.

\subsection{Regional effective duration of observations}

In a regional context, the effective duration of observations $D_{\text {eff }}$ of the regional sample depends on those of local samples $d_{i}(i=1, \ldots N)$, where $N$ is the number of sites in the region of interest. Indeed, $D_{\text {eff }}$ must be filtered of any intersite or any spatial dependencies and correlations. If on-site samples in a given region are completely independent, pooling data from these sites leads to a regional effective duration of observations equal to $\sum d_{i}$. This is obviously not the case herein. In those cases where the intersite dependence is perfect, $D_{\text {eff }}$ can be formulated as the average of durations of observations $d_{i}$. Weiss et al. (2014) have developed a spatial function $\varphi$ that reflects the intersite dependence in a region of interest. Weiss et al. (2014) have shown that $\varphi=\lambda_{\mathrm{r}} / \lambda$, where $\lambda_{\mathrm{r}}$ is the average annual number of storms in the region and $\lambda$ is the average number of storms per year at each site. $D_{\text {eff }}$ is expressed as a weighted sum in the following form:

$D_{\text {eff }}=\frac{\varphi}{N} \sum_{1}^{N} d_{i}=\frac{n_{\mathrm{r}}}{\lambda}$,

where $n_{\mathrm{r}}$ is the number of regional SSSs. The function $\varphi$ takes a value between 1 and $N$ according to the level of dependence in the region.

$-\varphi=1$ if the region is perfectly regionally dependent. The effective duration of observations $D_{\text {eff }}$ takes then the form of the average of durations: $D_{\text {eff }}=1 / N \sum d_{i}$.

$-\varphi=N$ if the region is regionally independent. This leads to $D_{\text {eff }}=\sum d_{i}$.

\subsection{Fitting the regional SSSs and at target-site frequency estimations}

The regional frequency model used herein is based on the extreme value theory. As mentioned in Sect. 2.2, the peaksover-threshold (POT) approach (e.g., Pickands, 1975), in which the excesses are analyzed with the GPD, is used in the RFA, using a threshold equal to 1 and taking into account the seasonality. Seasonal effects, considered for other variables in the literature (e.g., Morton et al., 1997; Méndez et al., 2008), can be modeled through a sinusoid. The regional distribution becomes a discrete mixture of GPD and sinusoid, with a seasonally varying scale parameter $\xi$ ( $\xi$ varies periodically and smoothly across the seasons) (Weiss, 2014). The probability distributions are constructed as follows.

Let us denote $v$ the regional random variable. A GPD is fitted to the regional sample, taking into account the four seasons in the following way: 
$\forall v \geq 1, F_{\mathrm{r}}(v)=\sum_{c=1}^{4} p_{\mathrm{r}, \mathrm{c}} F_{\mathrm{r}, \mathrm{c}}(v)$

$p_{\mathrm{r}, \mathrm{c}}$ is the frequency of occurrence of season $c$ in the regional sample (empirically estimated as the observed proportion of storms that occurred during season $c$ in the region). We also have

$F_{\mathrm{r}, \mathrm{c}} \sim \operatorname{GPD}\left(1, \gamma_{\mathrm{r}, \mathrm{c}} k_{\mathrm{r}}\right)$,

and

$\log \left(\gamma_{\mathrm{r}, \mathrm{c}}\right)=\gamma_{\mathrm{r}}^{0}+\gamma_{\mathrm{r}}^{1} \cos \left(\frac{2 \pi}{4} c\right)+\gamma_{\mathrm{r}}^{2} \sin \left(\frac{2 \pi}{4} c\right)$,

where $\gamma_{\mathrm{r}}^{0}, \gamma_{\mathrm{r}}^{1}$, and $\gamma_{\mathrm{r}}^{2}$ are three parameters to be estimated. Table 3 presents the eight models derived from the possible values of $\gamma_{\mathrm{r}}^{0}, \gamma_{\mathrm{r}}^{1}, \gamma_{\mathrm{r}}^{2}$, and $k_{\mathrm{r}}$. Besides the visual inspection of the fitting curve, the Akaike information criterion (AIC) is used to select the more adequate distribution. Finally, the frequency estimation can be easily reverted to estimate the on-target-site SSRLs by multiplying the regional ones by the local index (i.e., the annual SSS quantile).

\subsection{The case study}

SSS datasets are obtained from the temporal series of hourly observations and predicted tide levels (the astronomical tide), collected at a total of 67 sites located on the Spanish, French (Atlantic and English Channel), and British coasts (see Fig. 2). The French tide gauges are managed by the French oceanographic service (SHOM - Service Hydrographique et Océanographique de la Marine), while Spanish and British ones are managed by IEO (Instituto Español de Oceanografía, Spain) and the British Oceanographic Data Centre (BODC), respectively.

For convenience, the same observation periods as those used by Weiss (2014) were used in the present study. They range from 1846 for Brest (in France) to 2011 (for almost all the sites), and they show an average effective duration of observations of 31 years. In most cases, local series are characterized by the presence of many gaps. It is to be noted that the sea levels must be corrected from a possible eustatism (a general variation in mean sea level) in order to avoid inducing bias in the calculation of the surges: a correction is done if annual sea levels (calculated following the Permanent Service for Mean Sea Level recommendations) show significant trends. It is also noteworthy that the impact of climate change on the estimated return levels and associated uncertainties is not covered by this paper. The use of projected sea level rise could, however, be the subject of another paper.

Furthermore, in connection with the choice of the variable of interest, the focus is restricted to SSS series because in regions with strong tidal influence, the coastal flooding hazard is most noticeable around the time of high tide. Indeed, the
SSS is a fundamental input for many statistical investigations of coastal hazards. It is defined as the difference between the maximum observed sea level and the one predicted around the time of high tide. Thus, the resulting SSS series have a temporal resolution of approximately $12.4 \mathrm{~h}$. The reader is referred to Bernardara et al. (2011) for a more detailed introduction on skew surges. The developed RFA is performed at many target sites along the French (Atlantic and English Channel) coast. One of the most important features of these target sites is the fact that the region in which they are located has experienced significant storms during the last few decades (1953, 1987, Lothar and Martin in 1999, and Xynthia in 2010). Figure 2 displays the geographic location of the whole region. As depicted in the left-hand side of the figure, three target sites (red empty circles) are selected to perform the developed methodology and estimate the 1000-year return level.

\section{Results}

All the simulations are carried out within the R environment (open-source software for statistical computing: http://www. r-project.org/, last access: 9 June 2020). The main results of the developed RFA with all the diagnostics are presented in terms of tables and figures (probability plots), wherein the main focus is set on the storm surge quantile corresponding to the return period $T=1000$ years and the width of the $70 \%$ confidence interval (CI). Prior to the RFA, the results of the homogenous region delineation are presented first.

\subsection{Formation of regions of interest centered on target sites}

Two types of thresholds are used in the calculation of the empirical spatial extremogram. The first threshold sets the extreme quantiles to extract extreme SSSs, and the second one (the neighborhood threshold) sets the extremal coefficient above which sites are considered neighbors. Since thresholds that are too high result in introducing a high variance and those that are too low introduce a bias in the results, there is a trade-off to be made between variance and bias. Indeed, the asymptotic properties of the marginal SSSs can be violated when too low of an extreme quantile $q_{\mathrm{p}}(p<70 \%$, for instance) is used to compute the extremal dependence coefficient of a given site. The use of too high of a threshold ( $p>99 \%$, for instance) can significantly reduce the number of the pairs of simultaneous extreme events to be used to compute the extremal coefficient. It is to be noted that, even if the $q_{\mathrm{p}}$ threshold is adequately selected, too high of a neighborhood threshold ( $\rho_{0}>0.7$, for instance) will limit the number of neighboring sites and decrease the size of the region of interest, while too low of a threshold will likely cause a residual probability (which is nothing other than a noise) and will erroneously increase this region. Values of $q_{X}$ 


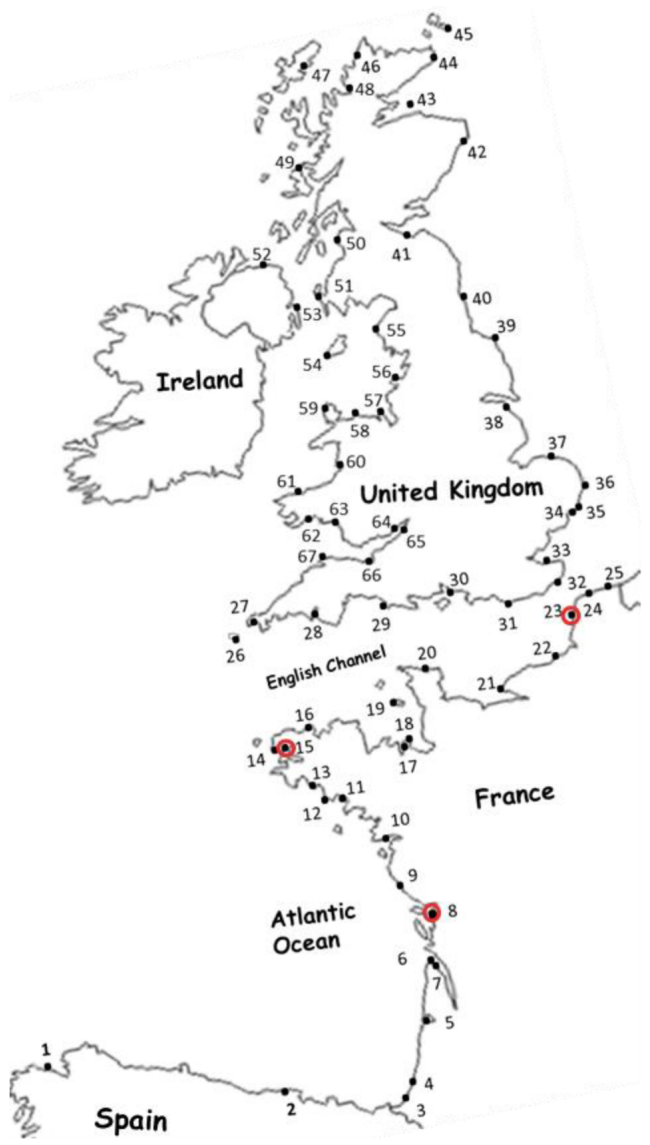

\begin{tabular}{ll}
\hline \multicolumn{2}{c}{ Sites } \\
\hline 1. La Coruna & 35. Felixstowe \\
2. Bayonne & 36. Lowestoft \\
3. St-Jean & 37. Cromer \\
4. Bayonne & 38. Immingham \\
5. Arcachon & 39. Whitby \\
6. Port Bloc & 40. North Shields \\
7. Verdon & 41. Leith \\
8. La Rochelle & 42. Aberdeen \\
9. Olonne & 43. Moray Firth \\
10. St-Nazaire & 44. Wick \\
11. Le Crouesty & 45. Lerwick \\
12. Port Tudy & 46. Kinlochbervie \\
13. Concarneau & 47. Stornoway \\
14. Le Conquet & 48. Ullapool \\
15. Brest & 49. Stornoway \\
16. Roscoff & 50. Millport \\
17. Servan & 51. Portpatrick \\
18. St-Malo & 52. Portrush \\
19. Jersey & 53. Bangor \\
20. Cherbourg & 54. Port Erin \\
21. Le Havre & 55. Workington \\
22. Dieppe & 56. Heysham \\
23. Boulogne & 57. Liverpool \\
24. Calais & 58. Llandudno \\
25. Dunkerque & 59. Holyhead \\
26. St-Mary & 60. Barmouth \\
27. Newlyn & 61. Fishguard \\
28. Devonport & 62. Milford Haven \\
29. Weymouth & 63. Mumbles \\
30. Portsmouth & 64. New Port \\
31. Newhaven & 65. Avonmouth \\
32. Dover & 66. Hinkley Point \\
33. Sheerness & 67. Ilfracombe \\
34. Harwich & \\
&
\end{tabular}

Figure 2. Location of sites used for the study: 67 ports along the Spanish, French, and British coasts. Each site is associated with a number. The table on the right shows the correspondence between numbers and sites. The circled points represent the target sites for which a centered RFA is carried out in this study.

and $q_{Y}$ are tested in order to select four and then six storms a year, which finally give information from the empirical spatial extremogram that leads to similar regions. Therefore, the extremal quantiles are set to select only four storms a year. This value allows for the computation of the empirical spatial extremogram from the biggest storm of each year. Moreover, the lag time $h$ has to be large enough to allow a storm which occurs at one site to propagate eventually to the other site. Spatial extremograms performed with lag times greater than $24 \mathrm{~h}$ show little difference compared to those obtained with lag times equal to zero and to the duration between two SSSs (about $\pm 12.4 \mathrm{~h}$ ). The latest two lag times are then used, and the greatest value among the corresponding extremal coefficients is kept. Finally, the neighborhood threshold is set to 0.3 . This value allows for the elimination of any sites associated with a value of the spatial extremal coefficient that look like a residual noise.

\subsubsection{Calais (station number 24)}

One of the most important features of the Calais site is the fact that it is located close to a border of one of the regions found by Weiss (2014). In addition, the region in which this site is located has experienced significant storms during the last 2 decades (Martin in 1999 and Xynthia in 2010). Figure 3 displays the geographic location of five homogeneous regions according to Weiss (2014). The scheme for obtaining the pairwise extremal dependence coefficients between Calais as a target site and all the other sites is applied herein. From the ESE depicted in the Fig. 4a, a geographically coherent region of interest corresponding to the neighborhood threshold $\left(\rho_{0}=0.3\right)$ is obtained and illustrated in the leftmost panel of Fig. 5. As can be seen in Fig. 4, the pairwise probabilities of the extremal dependence between Calais and all the sites in the whole region are presented on the vertical axis. The sites of the whole region, presented on the $x$ axis, are sorted in an ascending order based on the geographical distance to the target site. The physically homogeneous group of sites with extremal dependence probabilities greater than $\rho_{0}$ (the red lines on Fig. 4) are considered to be potential neighbors of the target site (Calais) and are thus part of the region of interest (of Calais). The pairwise simultaneous length of record (at the target site and any site of the whole region) appears in brackets next to the name of each site in Fig. 4. 


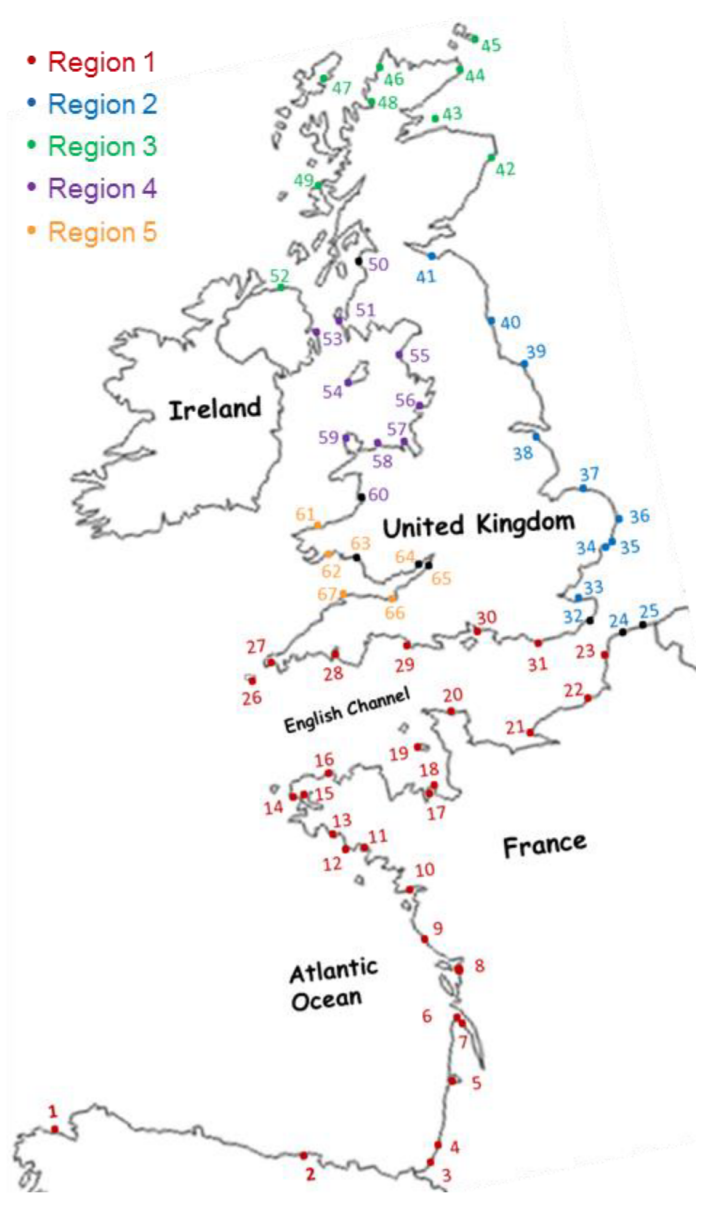

Figure 3. Five physically and statistically homogenous regions (according to Weiss, 2014). The regions are represented by five colors. This figure shows that, for example, site 24 (Calais) is located in the region shown in blue and is very close to a border. Site 23 (Boulogne), however, which is very close to site 24 (Calais), is nevertheless in another region (the region shown in red). This separation between site 23 and site 24 may seem artificial.

This duration is an important setting because it is the number of years on which the spatial extremal coefficients are calculated. For instance, the time during which Calais and Dunkerque (station number 25) operated simultaneously is equal to 26 years. It is noteworthy that the probability of the extremal dependence may not be relevant if this time period is too small, and whether it is appropriate to consider the site in the inference will be assessed on a case-by-case basis. As shown in the leftmost panel of Fig. 5, the target site Calais is no longer located at the border of the region. Indeed, the physically homogeneous region of interest around Calais is slightly smaller than the one obtained by Weiss (2014) but more centered on Calais. Further noteworthy features of the ESE are that it provides regions smaller than those obtained by Weiss (2014) and that are more physically homogeneous.

\subsubsection{Brest (station number 15)}

The ESE for the whole region with Brest as a target site is shown in Fig. 4b, and the associated region of interest is depicted in the middle panel of Fig. 5. As shown in this figure, the region of interest around Brest is larger than the one centered on Calais, with many sites for which the extremal dependence coefficients are at the limit of the neighboring threshold $\rho_{0}$. This is definitely the case for sites located in the Bristol Channel on the British coast. A sensitivity study is conducted with and without these sites, and it is concluded that these sites should remain in the region of interest. Indeed, their absence led to less adequate fitting. As mentioned earlier and as shown in the middle panel of Fig. 5, the homogeneous region centered on Brest is smaller than the one obtained by Weiss (2014), but it is nevertheless better centered on the target site Brest.

\subsubsection{La Rochelle (station number 8)}

One of the most important features of the La Rochelle site is the fact that the region in which this site is located recently experienced the storm Xynthia (2010). It has been the subject of many studies after this storm (e.g., Hamdi et al., 2015). Figure 4c shows the ESE (with La Rochelle as a target site), and in the rightmost panel of Fig. 5 the homogeneous region of interest centered on La Rochelle is depicted. As concluded with the two first target sites, Calais and Brest, it can be seen in the rightmost panel of Fig. 5 that the region of interest is also smaller than that obtained by Weiss (2014) but better centered on the La Rochelle site. The time during which La Rochelle and both Saint-Malo (station number 18) and Saint-Servan (station number 17) sites operated simultaneously is relatively small (14 years for Saint-Malo and 2 years for Saint-Servan). The extremal dependence coefficients for these two sites are equal to 0.29 and 0.4 , respectively. The question of whether to consider Saint-Servan and Saint-Malo as being inside the region or not has been raised. Since both sites are very close to each other (with a distance of less than $2 \mathrm{~km}$ ), it seems logical to either add them both to the region or withdraw them both from the region. We finally decided to integrate them into the region of interest because the site Jersey is part of the region centered on La Rochelle, with a dependency extremal probability equal to the neighboring threshold $\left(\rho_{0}=0.3\right)$ and a common period of only 14 years. The site Jersey, abbreviated "JER" in the ESE plots with the station number 19 , is geographically very close to Saint-Malo and Saint-Servan.

Once the physically homogeneous regions are formed, the statistical homogeneity must be verified. As mentioned earlier in this paper, the L-moment-based homogeneity tests (heterogeneity measure and discrepancy) are used. The heterogeneity measure $H$ is equal to -0.13 for Calais, 0.99 for Brest, and 1.13 for La Rochelle. The only case where there has been a discordant site is when La Rochelle was the target 
(a)

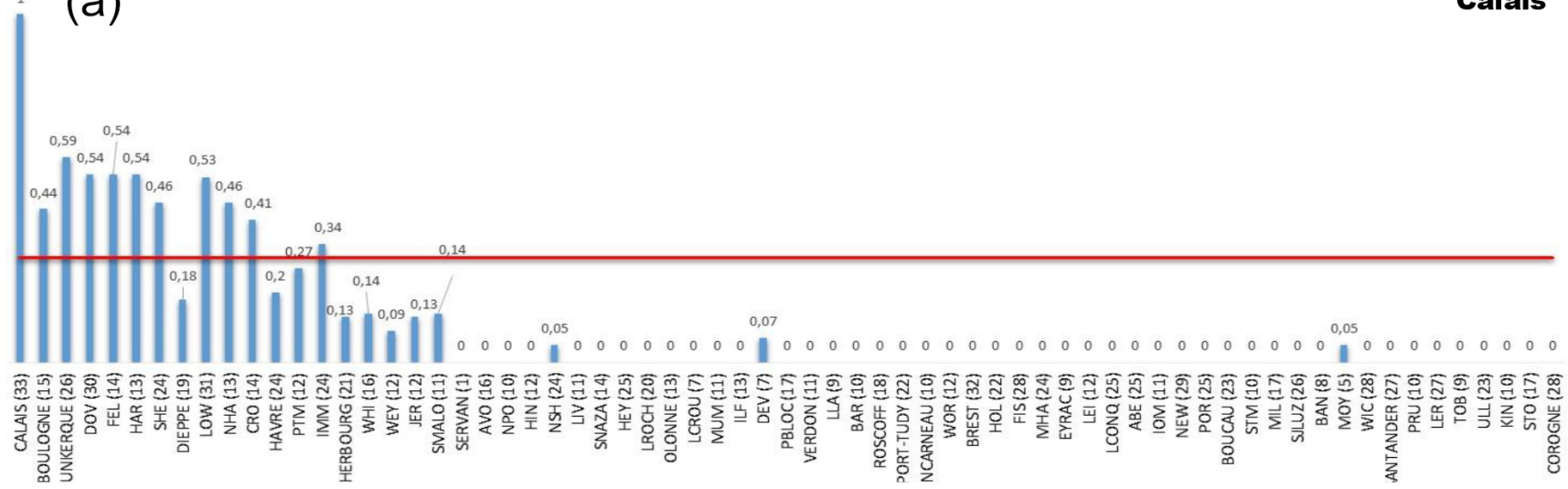

(b)

Brest
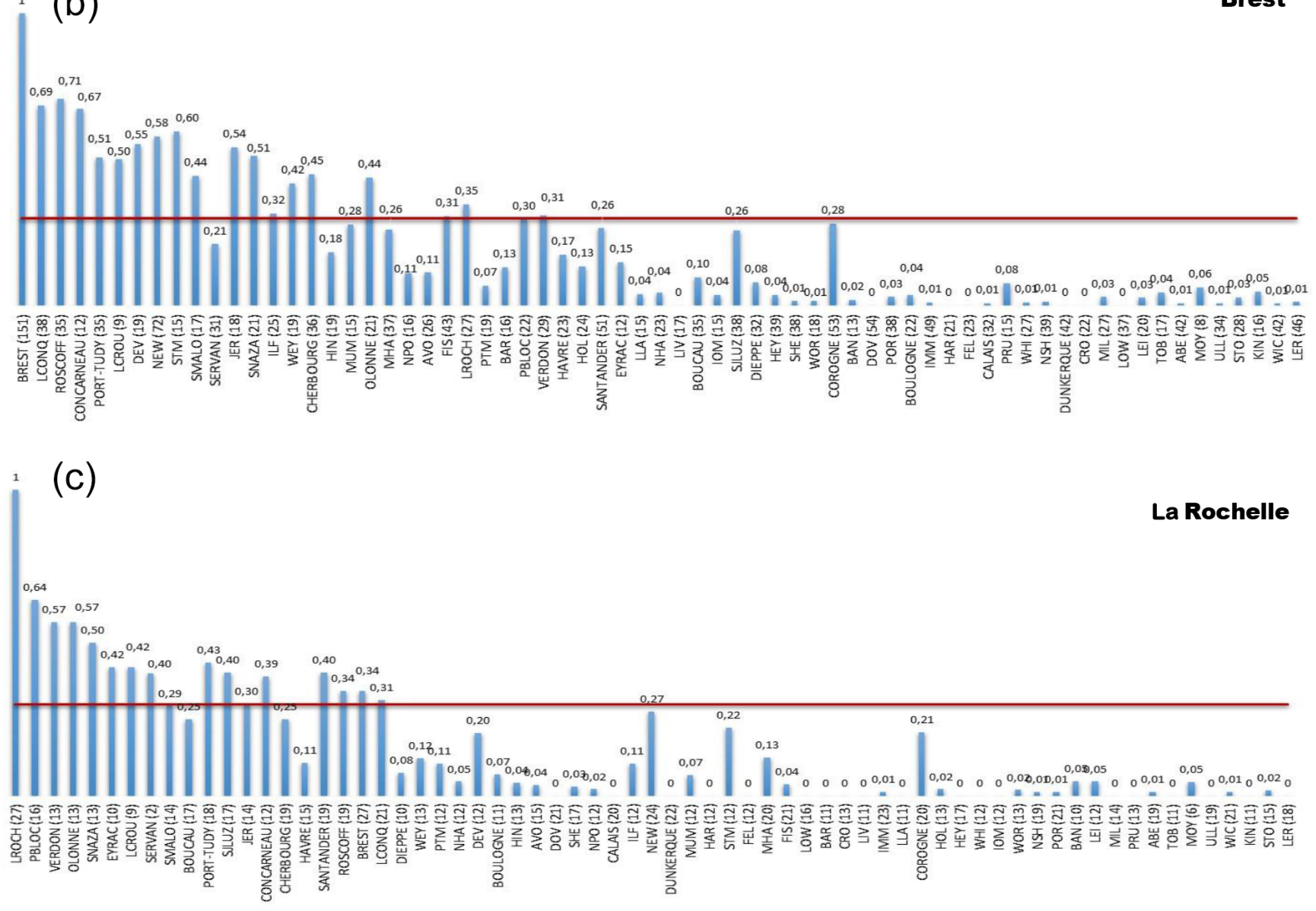

Figure 4. The ESE for Calais (a), Brest (b), and La Rochelle (c). The abbreviations of the horizontal axis are presented in Fig. 2. The vertical blue bars have lengths proportional to the extremogram values. The value of each extremogram is indicated above the blue bars. The red line represents the threshold (equal to 0.3 ) above which the extremogram value is large enough that the associated site can be considered to belong to the same region as the target site. The overlap period (in years) of the observation periods of each site with that of the target site is indicated in the brackets next to each site name. 

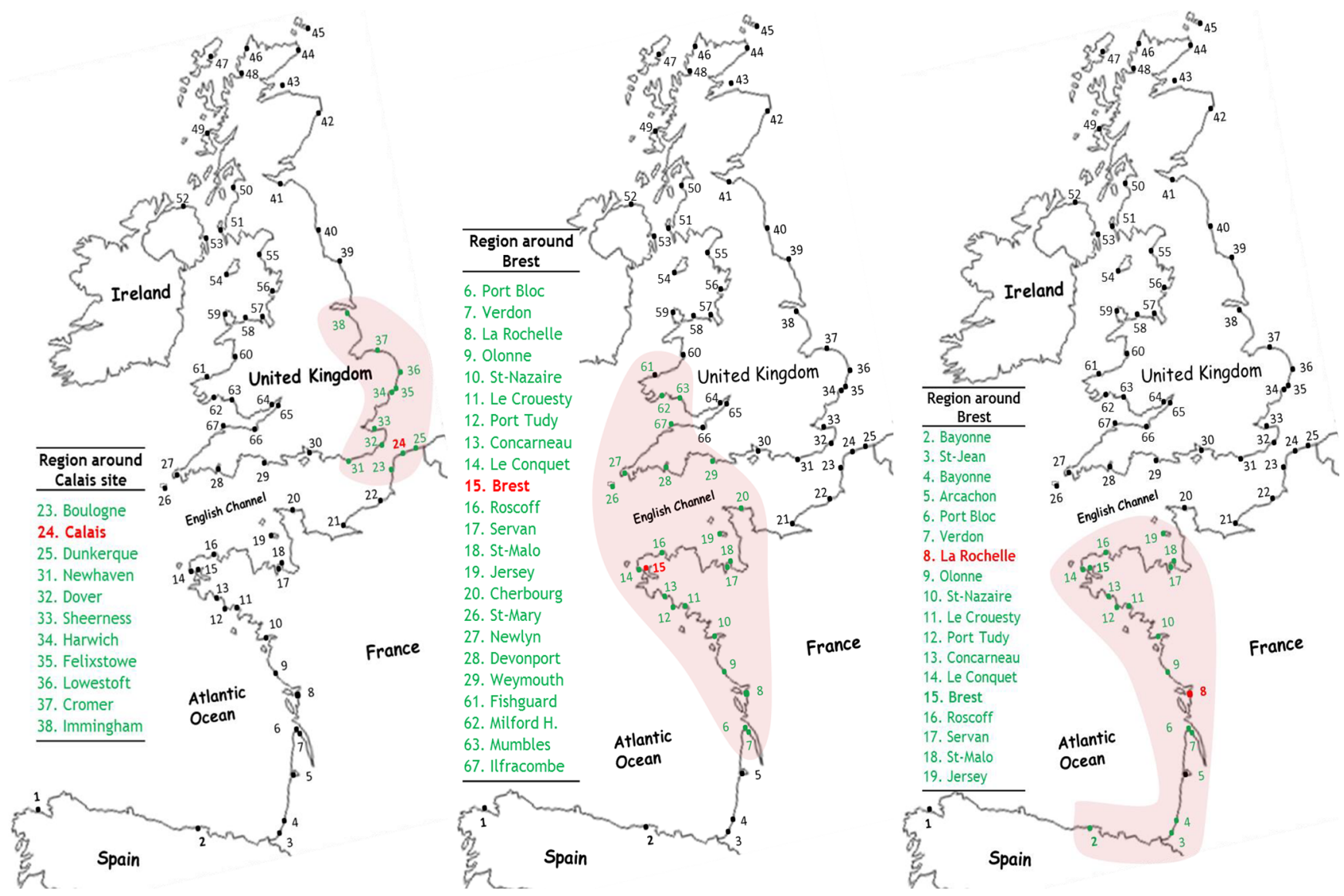

Figure 5. Physically homogenous regions (the list of sites belonging to a region are surrounded by red zones) for Calais, Brest, and La Rochelle. Neighboring sites are represented with green dots (target sites are represented with red dots). Target sites are not close to a border.

site. Indeed, with a $D_{\mathrm{c}}$ of 3.65 , the Eyrac site (station number 5) has been identified as discordant. This site is located in the center of the region, and this discrepancy could be explained by the specific sea conditions in the Arcachon basin. It is noteworthy that when Eyrac is removed from the region, the heterogeneity measure $H$ becomes equal to 0.53 . Thus a new region without Eyrac being considered statistically homogeneous is used.

\subsection{The regional and local frequency estimations}

As mentioned earlier in this paper, a regional pooling method to estimate the regional distribution for each homogeneous region is used. Indeed, a storm that can impact several sites (thus generating intersite dependence) during a single storm is considered only once in the regional sample. The distribution of the maximum regional SSSs $\left(M_{\mathrm{S}}\right)$ is assumed to be identical to the regional distribution. In order to verify the validity of this assumption, a Kolmogorov-Smirnov test ( $H_{\mathrm{o}}$ : the regional observations and $M_{\mathrm{s}}$ sample follow the same distribution) is performed. The null hypothesis $H_{\mathrm{o}}$ is satisfied for the three regions centered on Calais, Brest, and
La Rochelle at a risk level of $5 \%$. Consequently, the regional distribution can be estimated from each regional sample $M_{\mathrm{s}}$. The way the delineation of the homogeneous regions is performed implies that the regional sample is characterized by a strong spatial dependence which impacts, in a first step, the regional effective duration of observations $D_{\text {eff }}$ (that will be even lower if this dependence is high). The effective duration of observations $D_{\text {eff }}$ is calculated using Eq. (3), and the results are compared with those obtained by Weiss (2014). These results are summarized in Table 1. It is important to remember here that in the study of Weiss (2014), Calais is a part of region 2, while Brest and La Rochelle are located in region 1. As shown in Table 1, the regional effective duration of observations $D_{\text {eff }}$ associated with the region of Calais is the same in both studies. However, it is smaller in the present work for the region of Brest and La Rochelle. In any case, however, regional effective durations are obviously higher than local ones. Furthermore, a Student $t$ test is performed to check if the regional sample is stationary in intensity (two subsamples have equal means) or not. It is concluded that all the samples are stationary in intensity. 
Table 1. Comparison of total and effective durations of observations (years) used in the present study with those used by Weiss (2014). The effective duration of observations takes into account the intersite dependence. A total duration of observations is the sum of all on-site durations in the region of interest (without intersite dependence).

\begin{tabular}{lccccccc}
\hline & \multicolumn{3}{c}{ The present work } & & \multicolumn{2}{c}{ Weiss (2014) } \\
\cline { 2 - 3 } \cline { 6 - 7 } & Calais & Brest & $\begin{array}{c}\text { La } \\
\text { Rochelle }\end{array}$ & & $\begin{array}{c}\text { Region 1 } \\
\text { (Calais) }\end{array}$ & $\begin{array}{c}\text { Region 2 } \\
\text { (Brest and } \\
\text { La Rochelle) }\end{array}$ \\
\hline$D_{\text {eff }}$ & 151 & 348 & 348 & & 517 & 151 \\
Total duration & 375 & 783 & 605 & & 1011 & 443 \\
\hline
\end{tabular}

Table 2. Comparison of the 1000-year return levels with the $70 \%$ of the confidence interval width $(70 \% \mathrm{CI})$ in brackets obtained herein with those of Weiss (2014).

\begin{tabular}{lccc}
\hline & \multicolumn{3}{c}{1000 -year RLs (70 \% CI) (m) } \\
\cline { 2 - 4 } & Calais & Brest & La Rochelle \\
\hline Results from present study & $1.55(0.18)$ & $1.68(0.19)$ & $1.68(0.17)$ \\
Results from Weiss (2014) & $1.62(0.30)$ & $1.56(0.14)$ & $1.67(0.15)$ \\
\hline
\end{tabular}

A GPD distribution taking into account the seasonality is then fitted to the regional sample. The distribution parameters are estimated with the penalized maximum likelihood method (Coles and Dixon, 1999). The most adequate distributions are obtained with the AIC. The $\operatorname{Exp}_{\text {sin }}$ distribution is selected for Calais, while it is concluded that the distributions of the extreme regional SSSs for Brest and La Rochelle converge to a $\mathrm{Gpd}_{\mathrm{cos}} \sin$. The same frequency models are selected by Weiss (2014) for regions 2 (including Calais) and 1 (including Brest and La Rochelle), respectively. The reader is referred to Weiss (2014) to learn more about the mixture of GPD-sinusoid distributions with a seasonally varying scale parameter. The fitting curves for Calais, Brest, and La Rochelle, which are shown in Fig. 6, look good. Indeed, most of the points in the body of the distribution are inside the confidence intervals. Those elements are also relevant for accepting the credibility of the distributions used for the fitting.

In the next RFA step, local quantiles are estimated by multiplying the regional ones by the local indices. The results are summarized in Table 2, presenting a comparison of the 1000year return levels with associated confidence intervals.

It is worth concluding that better centering the region of interest on the Calais site did not significantly change quantiles (a decrease of only $7 \mathrm{~cm}$ ) but rather narrowed the associated confidence interval of about $12 \mathrm{~cm}$. This outcome refers only to the region of interest around Calais, and it is different for the region centered on Brest and La Rochelle sites. However, the quantiles and associated confidence intervals are overall roughly the same, but the method presented herein better answers the uncertainties linked to the border effect issue, notably through the ESE tool.
Table 3. Possible models for the fitting of the regional samples.

\begin{tabular}{ll}
\hline Model names & Parameter values \\
\hline $\operatorname{Exp}$ & $\gamma_{\mathrm{r}}^{1}=\gamma_{\mathrm{r}}^{2}=k_{\mathrm{r}}=0, \gamma_{\mathrm{r}}^{0} \epsilon R$ \\
$\operatorname{Exp}_{\cos }$ & $\gamma_{\mathrm{r}}^{2}=k_{\mathrm{r}}=0,\left(\gamma_{\mathrm{r}}^{0} \gamma_{\mathrm{r}}^{1}\right) \epsilon R^{2}$ \\
$\operatorname{Exp}_{\text {sin }}$ & $\gamma_{\mathrm{r}}^{1}=k_{\mathrm{r}}=0,\left(\gamma_{\mathrm{r}}^{0} \gamma_{\mathrm{r}}^{2}\right) \epsilon R^{2}$ \\
$\operatorname{Exp}_{\cos \sin }$ & $k_{\mathrm{r}}=0,\left(\gamma_{\mathrm{r}}^{0} \gamma_{\mathrm{r}}^{1} \gamma_{\mathrm{r}}^{2}\right) \epsilon R^{3}$ \\
$\mathrm{GPD}$ & $\gamma_{\mathrm{r}}^{1}=\gamma_{\mathrm{r}}^{2}=0,\left(\gamma_{\mathrm{r}}^{0} k_{\mathrm{r}}\right) \epsilon R^{2}$ \\
$\mathrm{GPD}_{\cos }$ & $\gamma_{\mathrm{r}}^{2}=0,\left(\gamma_{\mathrm{r}}^{0} \gamma_{\mathrm{r}}^{1} k_{\mathrm{r}}\right) \epsilon R^{3}$ \\
$\mathrm{GPD}_{\sin }$ & $\gamma_{\mathrm{r}}^{1}=0,\left(\gamma_{\mathrm{r}}^{0} \gamma_{\mathrm{r}}^{2} k_{\mathrm{r}}\right) \epsilon R^{3}$ \\
$\mathrm{GPD}_{\cos \sin }$ & $\left(\gamma_{\mathrm{r}}^{0} \gamma_{\mathrm{r}}^{1} \gamma_{\mathrm{r}}^{2} k_{\mathrm{r}}\right) \epsilon R^{4}$ \\
\hline
\end{tabular}

\section{Discussion}

One of the most important features of the ESE-based approach used in this paper to form a physically homogenous region centered on a target site is the fact that it avoids the problem of the so called "border effect". Moreover, and in contrast to that introduced by Weiss (2014), the extremogram tool seems to prevent sites that are too distant from belonging to the same homogeneous region. This reduces physical and statistical heterogeneity that could be generated by pairwise sites that are quite far apart. Consequently, the spatial extremogram approach offers the key advantage leading to a certain geographical consistency. Despite the fact that the 1000-year return level and associated confidence interval obtained in this work are close to those obtained by Weiss (2014), the spatial extremogram method improves the physical homogeneity of the regions of interest and can decrease the effective duration of observations. Nevertheless, findings for the sites of Calais (which is no longer close to the border of a region) and Dunkerque seem to be particularly interesting for us because they are no longer close to the border of a region and since they can be representative sites for the Gravelines Nuclear Power Station in France. Furthermore, physical homogeneity may have an impact on the statistical one. Indeed, by using the L-moment-based criteria (Hosking and Wallis, 1997), it is concluded that unlike 

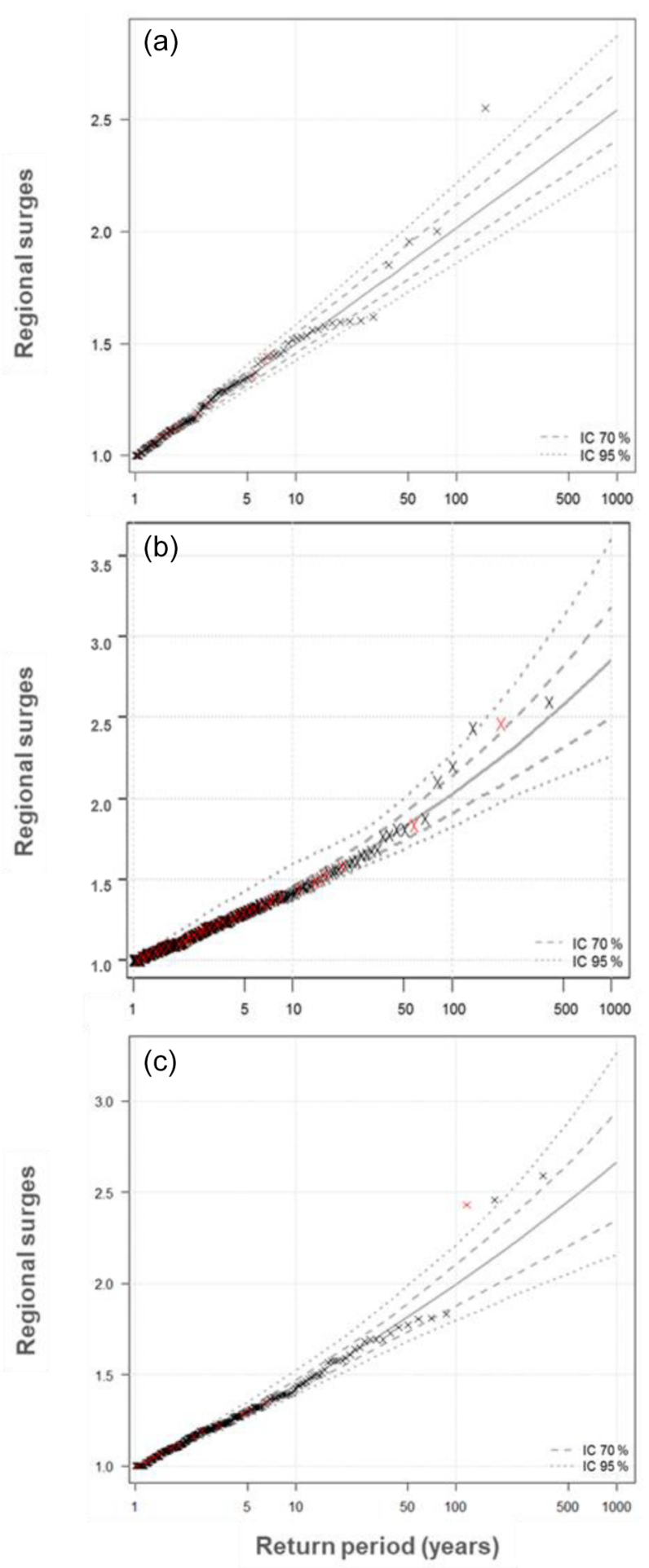

Figure 6. The GPD-sinusoid fitted to regional SSSs (plotting positions, RLs, and confidence intervals) for the target sites: Exp $_{\text {sin }}$ distribution for Calais (a) and GPD $\mathrm{Cos}_{\sin }$ for Brest (b) and La Rochelle (c). The $y$ axis represents the normalized regional surges (without unit); the $x$ axis represents the return period (in years). The red crosses indicate the SSSs at the target site, so the black crosses indicate the regional ones. The confidence intervals at $70 \%$ (dashed line) and $95 \%$ (dotted line), which are computed by the bootstrap method, are also presented (dotted lines). regions 1 and 2 in Weiss (2014) (which are considered to be possibly statistically homogeneous), all the regions built herein are statistically homogeneous, which is also progress.

The ESE-based approach can, nevertheless, be limited by the size of the common pairwise time period (during which data are present on both sites). Indeed, when the tide gauge at two different sites is often not operational over different periods of time, the common time period between these two sites used to calculate the spatial extremal coefficient may be short. Thus, sometimes a spatial extremogram can be considered not relevant, and therefore this shortcoming must be taken into account during the formation of the regions of interest, for instance by possibly removing the site involved. It will thus be interesting to analyze the uncertainties related to the ESE approach in order to have more reliability on the estimates of the extremal dependence between sites.

\section{Conclusions}

This study aims to perform regional frequency estimations of SSSs as an alternative to the local frequency analysis. Several ideas and approaches have been proposed in the literature to tackle the issue of the delineation of homogeneous regions, which is a main step in a RFA. The present work provides detailed reasoning for the need to use a more robust and reliable method which allows for the delineation of one homogeneous region centered on a target site and utilizes a method based on the calculation of pairwise extremal dependence coefficients (the empirical spatial extremogram) introduced by Hamdi et al. (2016) and compares the results with those obtained by Weiss (2014). The regional sample of the independent maximum normalized SSSs is then constructed from the series of the concordant sites in the region of interest. A regional effective duration of observations reflecting the intersite dependence of this sample is subsequently calculated and used in the regional frequency estimations.

Another consideration in this paper is applying and illustrating the ESE approach to a whole region containing sites located on the Spanish, French (Atlantic and English Channel), and British coasts with three target sites in France (Calais, Brest, and La Rochelle). A regional mixture of GPD-sinusoid distribution with a seasonally varying scale parameter and confidence intervals is examined. Overall, the results suggest that the regional analysis can be helpful in making a more appropriate assessment of the risk associated with the coastal flooding hazard. The application demonstrates also that the return levels (RLs) and associated confidence intervals estimates for Calais, Brest, and La Rochelle target sites are close to those obtained in a previous work (Weiss, 2014).

An in-depth study using more physical data and criteria in addition to the ESE (such as the atmospheric pressure or the wind speed and direction) could help to form regions that are more physically homogeneous. The concept of the ESE 
should find additional applications for the assessment of risk associated with other hazards in other climate and geoscience fields (e.g., extreme temperature and heatwave hazards). Associating confidence intervals with the spatial extremal coefficients could also be interesting. Another possible future endeavor is to perform a RFA using a regional sample containing all the regional SSSs (not only the maximum per storm and without considering the intersite dependence).

Data availability. The systematic data can be obtained by request addressed to the corresponding author: SHOM (Service Hydrographique et Océanographique de la Marine) for French data, the BODC (British Oceanographic Data Centre) for British data, and the IEO (Instituto Español de Oceanografía) for Spanish data.

Author contributions. Initially, MA and $\mathrm{YH}$ agreed at the same time on the idea of carrying out a centered regional frequency analysis of skew storm surge. SG programed the R code for the extremogram calculations from an idea of $\mathrm{YH}$, which was supported by MA. MA followed and guided all of SG's work. These works were presented to $\mathrm{YH}, \mathrm{PB}$, and $\mathrm{RF}$, who analyzed them. Their views were taken into account in the study. MA wrote the first version of the paper. $\mathrm{YH}$ and MA made the various corrections necessary during the revision process. RF also added some corrections. The conclusion was developed from the analyses of MA, SG, YH, RF, and PB.

Competing interests. The authors declare that they have no conflict of interest.

Special issue statement. This article is part of the special issue "Advances in extreme value analysis and application to natural hazards". It is a result of the Advances in Extreme Value Analysis and application to Natural Hazard (EVAN), Paris, France, 1719 September 2019.

Acknowledgements. The permission to publish the results of this ongoing research study was granted by the EDF (Electricité De France). The results in this paper should, of course, be considered to be $R \& D$ exercises without any significance or embedded commitments for the real behavior of the EDF power facilities or its regulatory control and licensing. The authors would like to acknowledge the SHOM (Service Hydrographique et Océanographique de la Marine, France), the BODC (British Oceanographic Data Centre, UK), and the IEO (Instituto Español de Oceanografía, Spain) for providing the data used in this study.

Review statement. This paper was edited by Ira Didenkulova and reviewed by Andreas Sterl and one anonymous referee.

\section{References}

Acreman, M. C. and Wiltshire, S. E.: Identification of regions for regional flood frequency analysis, Eos Trans. AGU, 68, 1262, 1987.

Bardet, L., Duluc, C.-M., Rebour, V., and L'Her, J.: Regional frequency analysis of extreme storm surges along the French coast, Nat. Hazards Earth Syst. Sci., 11, 1627-1639, https://doi.org/10.5194/nhess-11-1627-2011, 2011.

Bernardara, P., Andreewsky, M., and Benoit, M.: Application of the Regional Frequency Analysis to the estimation of extreme storm surges, J. Geophys. Res., 116, C02008, https://doi.org/10.1029/2010JC006229, 2011.

Burn, D.: Evaluation of regional flood frequency analysis with a region of influence approach, Water Resour. Res., 26, 2257-2265, https://doi.org/10.1029/WR026i010p02257, 1990.

Chebana, F. and Ouarda, T. B. M. J.: Depth and homogeneity in regional flood frequency analysis, Water Resour. Res., 44, W11422, https://doi.org/10.1029/2007WR006771, 2008.

Coles, S. and Dixon, M.: Likelihood-based inference for extreme value models, Extremes, 2, 5-23, https://doi.org/10.1023/A:1009905222644, 1999.

Darlymple, T.: Flood Frequency Analysis, Water Supply Paper 1543-A, US Geological Survey, United States Government Printing Office, Washington, https://doi.org/10.3133/wsp1543A, 1960.

Das, S. and Cunnane, C.: Examination of homogeneity of selected Irish pooling groups, Hydrol. Earth Syst. Sci., 15, 819-830, https://doi.org/10.5194/hess-15-819-2011, 2011.

Gabriele, S. and Chiaravalloti, F.: Using the Meteorological Information for the Regional Rainfall Frequency Analysis - An Application to Sicily, Water Resour. Manage., 27, 1721-1735, https://doi.org/10.1007/s11269-012-0235-6, 2013.

GREHYS - Groupe de Recherche en Hydrologie Statistique: Presentation and review of some methods for regional flood frequency analysis, J. Hydrol., 186, 63-84, https://doi.org/10.1016/S0022-1694(96)03042-9, 1996a.

GREHYS - Groupe de Recherche en Hydrologie Statistique: Intercomparison of regional flood frequency procedures for Canadian rivers, J. Hydrol., 186, 85-103, https://doi.org/10.1016/S00221694(96)03043-0, 1996b.

Hamdi, Y., Bardet, L., Duluc, C.-M., and Rebour, V.: Use of historical information in extreme-surge frequency estimation: the case of marine flooding on the La Rochelle site in France, Nat. Hazards Earth Syst. Sci., 15, 1515-1531, https://doi.org/10.5194/nhess-15-1515-2015, 2015.

Hamdi, Y., Duluc, C.-M., Bardet, L., and Rebour, V.: Development of a target-site-based regional frequency model using historical information, in: Vol. 18, Geophysical Research Abstracts of the EGU General Assembly 2016 Vienna, Austria, EGU2016-8765, 2016.

Hosking, J. R. M. and Wallis, J. R.: Some statistics useful in regional frequency analysis, Water Resour. Res., 29, 271-282, https://doi.org/10.1029/92WR01980, 1993.

Hosking, J. R. M. and Wallis, J. R.: Regional Frequency Analysis. An approach based on Lmoments, Cambridge University Press, Cambridge, https://doi.org/10.1017/CBO9780511529443, 1997.

Méndez, F. J., Menéndez, M., Luceño, A., Medina, R., and Graham, N. E.: Seasonality and duration in extreme value distri- 
butions of significant wave height, Ocean Eng., 35, 131-138, https://doi.org/10.1016/j.oceaneng.2007.07.012, 2008.

Morton, I. D., Bowers, J., and Mould, G.: Estimating return period wave heights and wind speeds using a seasonal point process model, Coast. Eng., 31, 305-326, https://doi.org/10.1016/S03783839(97)00016-1, 1997.

Pickands, J.: Statistical inference using extreme order statistics, Ann. Stat., 3, 119-131, 1975.
Weiss, J.: Analyse régionale des aléas maritimes extrêmes, $\mathrm{PhD}$ dissertation, University of Paris-Est, Paris, France, available at: https://pastel.archives-ouvertes.fr/tel-01127291 (last access: 9 June 2020), 2014.

Weiss, J., Bernardara, P., and Benoit, M.: Modelling intersite dependence for regional frequency analysis of extreme marine events, Water Resour. Res., 50, 5926-5940, https://doi.org/10.1002/2014WR015391, 2014. 